\title{
Increasing the Efficiency of Image Results through Improved Image Retrieval by using Image Mining in Search Engines
}

\author{
Devesh Batra \\ Student, Stanford University
}

\author{
Pragya Verma \\ Member, IEEE
}

\begin{abstract}
With the growth of Internet and advances in storage technologies, spread and acquisition of images has become imperative. Despite unprecedented advances in data mining, search engines return results of image queries by mining the written text associated with the images rather than mining the images to provide suitable results as per the image query. Image mining isn't merely an extension of data mining to image domain. It is an interdisciplinary attempt that draws upon expertise in various fields of Computer Science, ranging from computer vision, image processing and image retrieval to data mining, machine learning, database, and artificial intelligence. In this paper, we will suggest how image mining can be successfully implemented and will also suggest possible future research in the said discipline.
\end{abstract}

\section{Keywords}

Image Mining, Image Retrieval, Classification, Recognition, Association Rule Mining, Machine Learning

\section{INTRODUCTION}

Due to increase in the use of images with the rapid advancement in technology, the need to improve the efficiency of image results through enhanced image retrieval becomes necessary. These acquired images if retrieved and analysed properly can reveal enormous amount of information to the users.

Image mining can be considered an extension to data mining in the image domain. The database used to store images is an image database in comparison to the relational database used to store data. [1]

Several fields such as computer vision, image processing, image retrieval, data mining, machine learning and artificial intelligence lay the foundation of image mining.

In Image mining, a low-level pixel presentation contained in a raw image or image sequence is efficiently and effectively processed into a high level spatial objects and relationships.[2]

Image mining systems, covers two broad applications which are Domain-Specific applications and General applications. Domain-specific applications consist of extracting the most relevant image features into a form suitable for mining whereas, the general applications consist of generating image patterns that will help in understanding the interaction between high level human perception of images and low level image features.
The paper is presented as follows: section II consists of the objectives and research issues associated with Image mining. Section III presents two proposed frameworks for the use of Image mining in search engines. Section IV describes the techniques used in image mining. Section V demonstrates the applicability of Image mining to real-world search queries. Section VI presents the conclusions of the findings presented in this paper.

\section{OBJECTIVES AND ISSUES RELATED TO IMAGE MINING}

Image Mining is used to enhance the image search results through improved image retrieval thereby increasing user understanding and analysis. It is employed in domain rich with images. Further, it is an interdisciplinary attempt that draws upon expertise in various fields of computer science ranging from computer vision, image processing and image retrieval to data mining, machine learning, database and artificial intelligence.[3]

The basic concept behind image mining is the extraction of implicit knowledge, image data relationship, or other patterns not explicitly stored in the images and in between images and other alphanumeric data.

Image mining may sound similar to computer vision and image processing but in reality, it is different from both. The main focus of image mining is the extraction of patterns from a collection of large images whereas, the main focus of computer vision and image processing is in understanding and/or extracting features from a single image.

Moreover, a clear distinction can be made in between image mining and pattern recognition. In pattern recognition the main objective is to recognise some specific patterns; whereas the aim of image mining is to generate all significant patterns without prior knowledge of what patterns may be present.

The image mining process can be described as follows: the images stored in an image database are pre-processed to improve the quality of the stored images. These improved quality images then go through a lot of transformation and feature extraction in order to generate important features from the images. Data mining techniques are then applied to these features to discover essential patterns. These patterns are then analysed to obtain the final knowledge which is applicable to many applications. The process described above is as shown in figure 1.[4] 


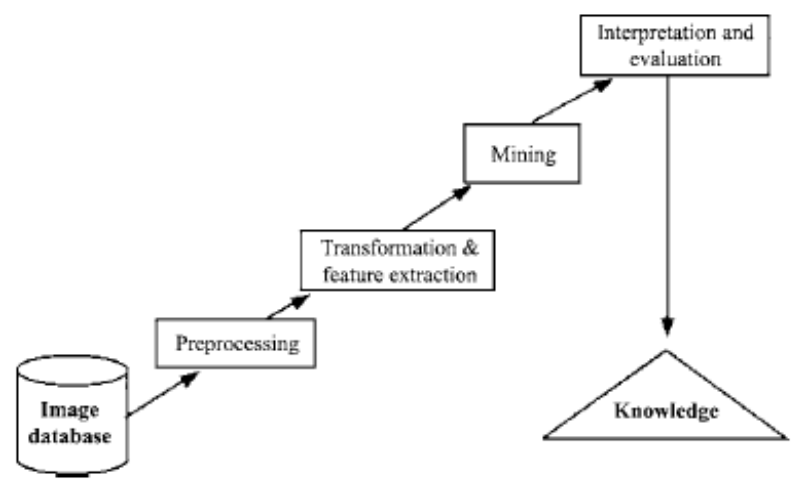

Figure 1: Image Mining Process

The database used for the purpose of storing images differs from the relational database which is used to store data in many ways. [5]Few of the most important differences are as follows:

1. In case of relational database the data stored has absolute values whereas the images stored in the image database has a relative value because images are dependent on the interpretation given by the viewer.

2. Implicit spatial information is essential for interpretation of image content but there is no such requirement in case of relational database.

\section{PROPOSED FRAMEWORK}

The images stored in the database are in a raw format and hence cannot be directly mined. In order to mine these images and obtain useful information from them we need to process these raw images.

An image mining system should provide various functionalities such as image storage, image processing, feature extraction, image indexing and retrieval, patterns and knowledge discovery.

An image mining system can be either based on functiondriven framework or information-driven framework Function-driven framework focuses on the functionality of different component modules whereas; the information-driven framework focuses on information available at different levels of hierarchy.

\subsection{Function-driven Framework}

A function-driven framework focuses on module functionality. It has four major components. These components are: (1) Image excavator for extraction of images. (2) Pre-processor for the extraction of image features and transforming raw images into images of better quality. (3) Search kernel for matching queries and (4) discovery modules to explore the underlying knowledge obtained from analysis of observed patterns in images.[7]

The architecture of function-driven framework is as shown in figure 2 .

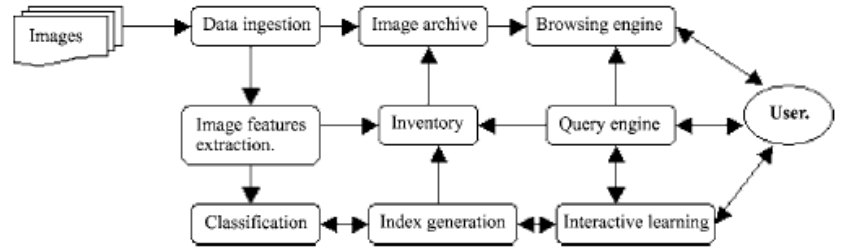

Figure 2: Architecture of function-driven framework

\subsection{Information-driven Framework}

Information-driven framework emphasises on representation of different levels of information which the Function-driven framework fails to provide.[8] The four basic levels of information represented by the information-driven framework are as follows:

1. Pixel Level: This level consists of raw image information such as image pixels and some basic image features for instance colour, texture, and shape.

2. Object Level: It deals with object or region information based on the primitive features obtained in the pixel level.

3. Semantic Concept Level: This level places the objects or regions identified in the previous levels in the context of the scenes depicted.

4. Pattern and Knowledge Level: This level integrates the domain related alphanumeric data and the semantic relationships discovered from the image data.

The first two levels are classified into lower layer whereas the last two levels fall into the category of higher layer. The information in the higher layer is more semantically meaningful than that present in the lower layer.[9] In other words, it can be said that the information contained in the lower layers is raw and the process of analysis needs to be applied to this raw information.

The architecture for information-driven Framework is as shown in figure 3.

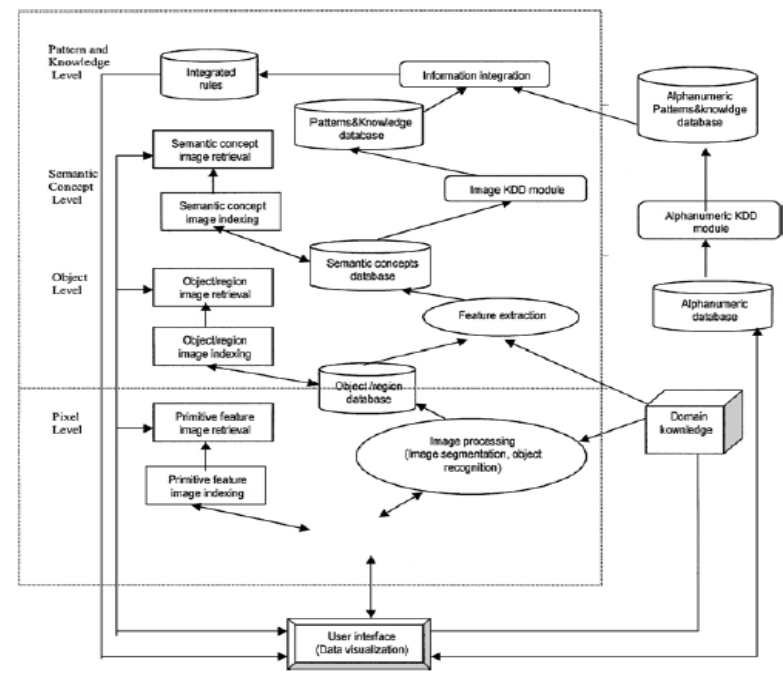

Figure 3: Architecture of Information-driven Framework 


\section{TECHNIQUES USED IN IMAGE MINING}

Few of the existing mining techniques used for extracting useful information can be extended to mining of images. These techniques include object recognition, image classification and clustering, association rule mining, artificial neural networks and image indexing and retrieval.[10]

These above mentioned techniques are explained in the following sub-sections.

\subsection{Object Recognition}

Using object models that are known a priori, an object recognition system finds objects in the real world from an image. [11]

During initial phases some objects need to be identified and recognised by a machine, this will lead to automatic machine learning and meaningful information extraction.

\subsection{Image Retrieval}

Retrieval of images can be classified into three levels of increasing complexity.

Level 1: Retrieval is done on the basis of primitive features such as colour, texture, shape or spatial location of image elements.

Level 2: Retrieval is done on the basis of derived or logical features like objects of a given type or individual objects or persons.

Level 3: Retrieval is done on the basis of abstract attributes involving significant amount of high-level reasoning about the meaning or purpose of the objects or scenes depicted.

Queries can be classified into three types namely, Query by Associate Attributes, Query by Description and Query by Image Content.

\subsection{Image Indexing}

High-Dimensional data is indexed, in order to reduce the search complexity.

A hybrid approach can also be employed, that is, both reduction of dimensions and use of multi-dimensional indexing.[12]

\subsection{Image Classification and Image Clustering}

Image classification and clustering are the supervised an unsupervised classifications of images into groups such that images belonging to one group are similar to each other.

In case of supervised classification, a collection of labelled images are given and a newly encountered unlabeled image is to be labelled. In unsupervised classification, the problem is to group a collection of unlabelled images into meaningful clusters according to the image content without a priori knowledge. Figure 4 shows the process of clustering.
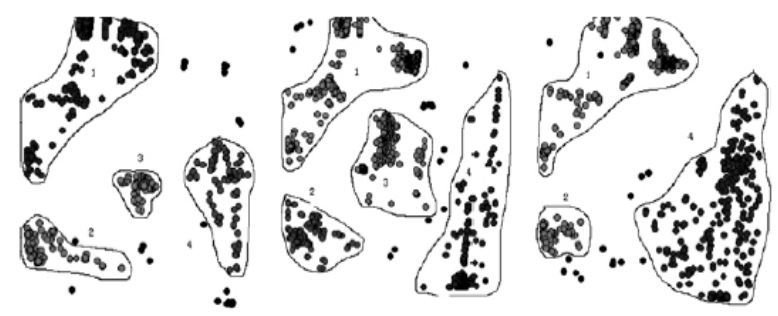

Figure 4: Image Clustering

\subsection{Association Rule Mining}

Association Rule mining is used to uncover interesting trends, patterns and rules in large datasets. Association Rule Mining employs one of the two approaches. The first approach is to mine from large collections of image alone and the second approach is to mine from a combine collection of images and associated alphanumeric data.[13]

\subsection{Artificial Neural Network}

Artificial Neural Networks can be defined in several ways. Neural networks can be defined as a class of mathematical algorithms. This definition is valid, since, a network can be regarded as a graphical notation for a large class of algorithms.[14] They can also be defined as synthetic networks that emulate the biological neural networks found in living organisms.

Application of artificial neural networks can be extended to the field of image mining as they are good in pattern recognition and trend prediction. They are frequently used to construct a model of the data.[15]

\section{APPLICABILITY OF IMAGE MINING TO REAL-WORLD SEARCH ENGINES}

In currently used search engines, the image search results are on the basis of text mining. The queries entered by the users are mined and an image which is associated with the mined text is displayed. The displayed image result may or may not be as per users' requirement and hence the need for image mining arises which will train the system to mine the image and show the appropriate search results.

Four queries were used to check the accuracy of image search results.

First, when a user types "park in front of the white house", out of the first 36 image search results shown, 21 were irrelevant. This can be demonstrated with the help of figure 5 .

Figure 5 shows a car in front of white house. The associated text with this image is WASHINGTON, D.C. - OCTOBER 17, 2001: MS rear of United States Park Police car parked in front of White House. The search engine performs text mining and mines the words park, front and white house and hence, this image though irrelevant is shown as a result of the search query. 


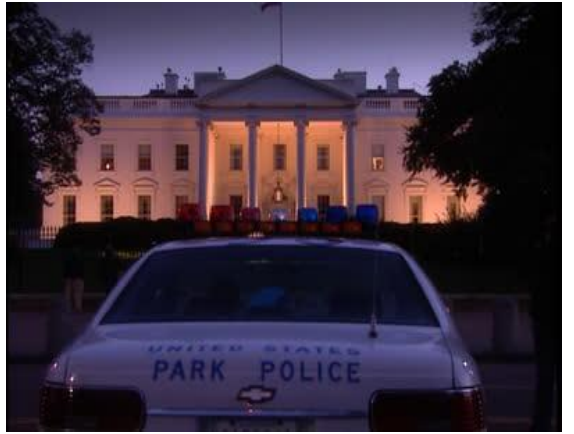

Figure 5: Park in front of White House

Second, when the user queries "red car in front of house", out of the first 36 image search results shown, 26 were irrelevant. This is explained with the help of figure 6 .

Figure 6 shows a non-red car in front of the house. The associated text with this image is 2006 Pontiac C6 GT Coupe Kelly's Car in Front of Our House Silver rsvr CL. The search engine performed text mining and the keywords it looked for were car, in front, house.

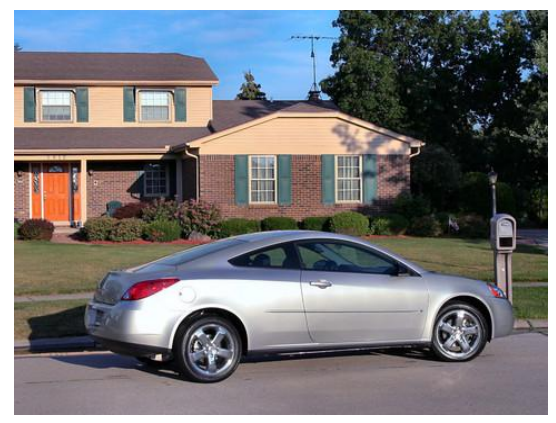

Figure 6: Red car in front of house

Third, the query entered was, "People sitting on road", out of the first 36 search results, 15 were irrelevant. Figure 7 illustrates one such example of an irrelevant picture.

The text associated with figure 7 is, Elephant causes West Midlands safari park traffic jam by sitting on road.

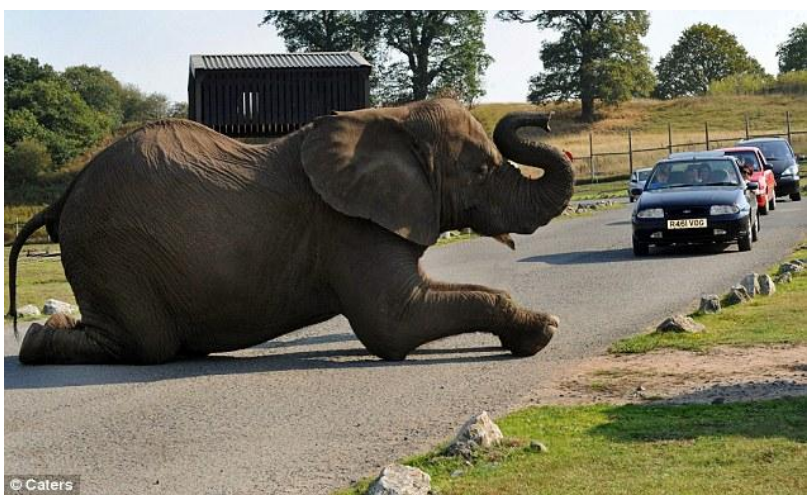

Figure 7: People sitting on road

Fourth, the query entered was "woman carrying black bag", out of the first 36 results shown, 30 were irrelevant. Figure 8 demonstrates one such example.

In figure 8 , the text associated with the image is, Black woman carrying reusable grocery bag.

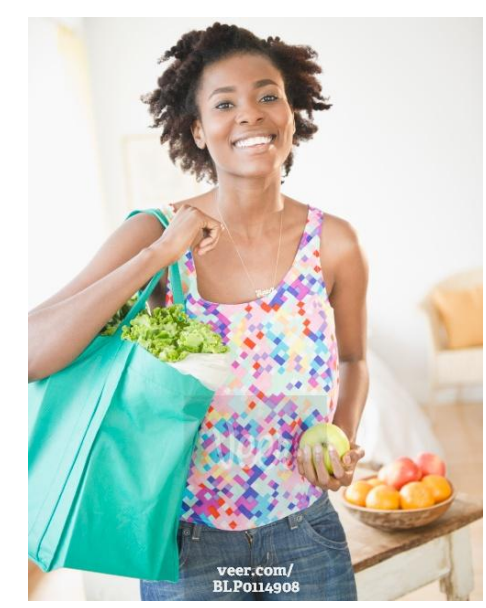

Figure 8: woman carrying black bag

The accuracy of the image search results obtained when the above mentioned four queries were run is shown in Table I.

Table I: Accuracy of Image search results

\begin{tabular}{|c|c|c|}
\hline Query Entered & $\begin{array}{c}\text { Number of } \\
\text { Relevant Images }\end{array}$ & Accuracy \\
\hline $\begin{array}{c}\text { Park in front of } \\
\text { the white house }\end{array}$ & 15 & $41.66 \%$ \\
\hline $\begin{array}{c}\text { Red car in front of } \\
\text { house }\end{array}$ & 10 & $27.77 \%$ \\
\hline $\begin{array}{c}\text { People sitting on } \\
\text { road }\end{array}$ & 21 & $58.33 \%$ \\
\hline $\begin{array}{c}\text { Woman carrying } \\
\text { black bag }\end{array}$ & 6 & $16.66 \%$ \\
\hline
\end{tabular}

The accuracy is calculated while keeping the total number of images in consideration to be fixed that is, 36 .

Since, the highest accuracy observed is $58.33 \%$, hence, the need of image mining arises. An image mining system will train the system with certain predefined images and later with the help of machine learning algorithms, appropriate image search results can be displayed to the user.

\section{CONCLUSION}

In this paper, we have highlighted the need of Image Mining in consideration to the swiftly increasing amount of Image data in use today. We have also explained the increase in efficiency of image results through improved image retrieval by using Image Mining in search engines. Further, we have described two frameworks which can be employed for the implementation of Image Mining in search engines. Various techniques used for mining are also discussed. Finally, the applicability of Image Mining to real-world search engines is also presented.

\section{REFERENCES}

[1] Antonie, Maria-Luiza, Osmar R. Zaiane, and Alexandru Coman. "Application of Data Mining Techniques for Medical Image Classification." MDM/KDD 2001 (2001): 94-101.

[2] Rui, Yong, Thomas S. Huang, and Shih-Fu Chang. "Image retrieval: Current techniques, promising directions, and open issues." Journal of visual communication and image representation 10.1 (1999): 39-62. 
[3] Bach, Jeffrey R., et al. "Virage image search engine: an open framework for image management." Electronic Imaging: Science \& Technology. International Society for Optics and Photonics, 1996.

[4] Bonet, J. S. D. "Image Preprocessing for Rapid Selection in "Pay Attention mode."." (2000).

[5] Bruzzone, Lorenzo, and Diego Fernàndez Prieto. "Unsupervised retraining of a maximum likelihood classifier for the analysis of multitemporal remote sensing images." Geoscience and Remote Sensing, IEEE Transactions on 39.2 (2001): 456-460.

[6] Cromp, Robert F., and William J. Campbell. "Data mining of multidimensional remotely sensed images." Proceedings of the second international conference on Information and knowledge management. ACM, 1993.

[7] Datcu, Mihai, and Klaus Seidel. "Image information mining: exploration of image content in large archives." Aerospace Conference Proceedings, 2000 IEEE. Vol. 3. IEEE, 2000.

[8] Fayyad, Usama M., et al. "Advances in knowledge discovery and data mining." (1996).

[9] Feder, Judy. "Towards image content-based retrieval for the World-Wide Web."Advanced imaging 11.1 (1996): 26-29.
[10] Gibson, Stephen, et al. "Intelligent mining in image databases, with applications to satellite imaging and to web search." Data mining and computational intelligence. Physica-Verlag HD, 2001. 309-336.

[11] Manjunath, Bangalore S., and Wei-Ying Ma. "Texture features for browsing and retrieval of image data." Pattern Analysis and Machine Intelligence, IEEE Transactions on 18.8 (1996): 837-842.

[12] Nastar, Chahab, et al. "Surfimage: a flexible contentbased image retrieval system." Proceedings of the sixth ACM international conference on Multimedia. ACM, 1998.

[13] Ordonez, Carlos, and Edward Omiecinski. "Discovering association rules based on image content." Research and Technology Advances in Digital Libraries, 1999. Proceedings. IEEE Forum on. IEEE, 1999.

[14] Chowdhury, Gobinda. Introduction to modern information retrieval. Facet publishing, 2010.

[15] Zhang, Ji, Wynne Hsu, and Mong Li Lee. "An information-driven framework for image mining." Database and expert systems applications. Springer Berlin Heidelberg, 2001 